\title{
Overlap between linear scleroderma, progressive facial hemiatrophy and immune-inflammatory encephalitis in a paediatric cohort
}

\author{
Lien De Somer ${ }^{1}$ - Marie-Anne Morren ${ }^{2}$ • P. C. E. Hissink Muller ${ }^{3} \cdot$ Karine Despontin $^{4}$. \\ Katrien Jansen $^{5} \cdot$ Lieven Lagae $^{5}$. Carine Wouters ${ }^{1}$
}

Received: 7 August 2014 / Revised: 19 March 2015 / Accepted: 24 March 2015

(C) Springer-Verlag Berlin Heidelberg 2015

\begin{abstract}
Linear scleroderma en coup the sabre (LSCS), progressive facial hemiatrophy (PFH) and autoimmune encephalitis are distinct clinical entities, although patients with overlapping features have been reported. We performed a multicenter retrospective review of a series of children with LSCS and/or PFH to explore the relation between these entities. The files of 16 children were reviewed, 11 presented with LSCS, 5 with PFH, with time overlapping cutaneous features were seen. Extracutaneous signs were found in both groups. ANA were present in more than $50 \%$ of patients. Almost half of our patients presented with CNS manifestations comprising unilateral headache, migraine and epilepsy with or without abnormalities on MRI. Brain biopsy in one patient was consistent with Rasmussen encephalitis. In two other children, associated autoimmune manifestations were present.

Conclusion: Our patient cohort brings more arguments to consider LSCS and PFH as a single disease entity with LSCS
\end{abstract}

and superficial skin involvement at one end of the spectrum and PFH with involvement of subcutaneous deep tissue at the other end. In both entities, encephalitis can be observed. Our findings of circulating ANA, intradermal lymphocytes and $\mathrm{IgG}$, intrathecal $\mathrm{IgG}$ production and clinical improvement with immunosuppressive therapy endorse the concept of a possible common immune-inflammatory pathogenesis.

What is known:

- LSCS, PFH and immune-inflammatory encephalitis are distinct clinical entities, but patients with overlapping features have been reported.

What is new:

- We present a unique paediatric cohort with LSCS, PFH and/or encephalitis.

- We endorse the concept of a common immune-inflammatory disease process.

Communicated by David Nadal

Revisions received: 24 October 2014/20 December 2014/09 March 2015

Lien De Somer

lien.desomer@uzleuven.be

Marie-Anne Morren

marie-anne.morren@uzleuven.be

P. C. E. Hissink Muller

P.C.E.Hissink_Muller@lumc.nl

Karine Despontin

despontin.karine@gmail.com

Katrien Jansen

Katrien.jansen@uzleuven.be

Lieven Lagae

lieven.lagae@uzleuven.be

Carine Wouters

carine.wouters@uzleuven.be

1 Department of Pediatric Rheumatology, University Hospital Leuven, Herestraat 49, 3000 Leuven, Belgium

2 Department of Pediatric Dermatology, University Hospital Leuven, Leuven, Belgium

3 Department of Pediatric Rheumatology, Leiden University Center, Leiden, The Netherlands

4 Department of Pediatric Dermatology, Centre Hospitalier du Luxembourg, Luxembourg, Luxembourg

5 Department of Pediatric Neurology, University Hospital Leuven, Leuven, Belgium 
Keywords Autoimmune encephalitis $\cdot$ Linear scleroderma en coup de sabre $\cdot$ Parry-Romberg syndrome $\cdot$ Progressive facial hemiatrophy

$\begin{array}{ll}\text { Abbreviations } \\ \text { ANA } & \text { Antinuclear antibodies } \\ \text { CSF } & \text { Cerebrospinal fluid } \\ \text { CNS } & \text { Central nervous system } \\ \text { EEG } & \text { Electroencephalography } \\ \text { MRI } & \text { Magnetic resonance imaging } \\ \text { LSCS } & \text { Linear scleroderma en coup the sabre } \\ \text { PFH } & \text { Progressive facial hemiatrophy } \\ \text { SPECT } & \text { Single photon emission computed tomography }\end{array}$

\section{Introduction}

Linear scleroderma is the most frequent form of localized scleroderma in children, involving $65 \%$ of patients in a large multicenter series [22]. The incidence in the general population is rare and estimated to 1 per 100,000. Involvement of the frontoparietal area is known as "en coup de sabre" (LSCS). The onset of this condition is mostly during late childhood, and stabilization occurs after several years. LSCS usually presents as a localized area of erythema or waxy induration with a surrounding halo of erythema. With time, the centre becomes shiny, ivory whitish and more infiltrated. It often leaves a hyperpigmented depressed scar. Lesions may extend to the scalp, where they cause alopecia, or to the nose, cheek, chin and neck. Atrophy of the affected side of the face is possible and usually develops within 1 year after onset. LSCS can occasionally be associated with central nervous system or ophthalmologic symptoms such as anterior uveitis, episcleritis, acquired glaucoma, xerophtalmia and keratitis [21].

Progressive facial hemiatrophy (PFH) or Parry-Romberg syndrome is a very rare condition that presents with severe atrophy of the soft tissues (without epidermal involvement) usually starting on the cheek, later extending to the forehead and scalp, sometimes resulting in alopecia, and to the chin or even the neck. The first signs usually appear in the first or second decade of life and a spontaneous stabilization occurs after 3-10 years, often with serious esthetical and neurological sequelae. Sclerosis of the skin is absent or less prominent than in linear scleroderma. The underlying bone and muscles may become atrophic leading to malocclusion of the jaws, teeth defects and asymmetric tongue. Central nervous system and ophthalmological complications are frequent $[15,21]$.

The hypothesis that LSCS and PFH are variants of the same disease process is a long-standing controversy [10, 15 , 18]. Moreover, overlap between these diseases and encephalitis has been reported $[1,13,14]$. The pathogenesis of these diseases remains a matter of discussion $[15,18]$.

With the present cohort study, we postulate to provide more arguments to the concept of an immune-inflammatory spectrum of disease that comprises these entities.

\section{Patients and methods}

The clinical records, imaging data and laboratory investigations of all children, diagnosed with LSCS or PFH, seen at the paediatric rheumatology, neurology or dermatology consultations of the Leuven University Hospital, Belgium, Centre Hospitalier Luxembourg and the Leiden University Center, the Netherlands, between 1988 and 2012 were reviewed.

A consent was obtained according to the Declaration of Helsinki; the study has been performed conforming the standards currently applied in Belgium, The Netherlands and Luxembourg. Photographs of patients were published with consent of the respective patients.

\section{Results}

After reviewing the medical files, 16 patients were included in this study. The clinical findings, laboratory results and imaging data are summarized in Table 1.

Eleven (11/16) patients presented with LSCS with a median age at presentation of 5 years (range 3-12 years). Five of these were male, 6 were female. Four of these patients developed atrophy of the hemiface, mostly within 1 to 2 years after appearance of the first signs of the initial lesion. The other five children (5/11) presented with PFH with a median age at presentation of 4.8 years (range 3-9, 5 years). The male/female ratio was 2:3. Most of them (3/5) had localized scleroderma lesions as well (Fig. 1). At the end of the observation period, nine of our patients (9/16) had obvious hemifacial atrophy.

In none of these children, there was a history of trauma or infection preceding the onset of the skin lesions.

Extracutaneous signs were found in 7 of the 11 patients with LSCS and in 4 of the 5 with PFH. Intra-oral lesions with asymmetry of teeth arches and missing teeth were present in 3 patients. Ophthalmologic abnormalities included ptosis of eyelid, enophthalmia, bilateral anterior uveitis and papillary asymmetry. CNS manifestations ranged from hemicranial headache over migraine to epilepsy and Rasmussen encephalitis. The 2 patients with seizures developed this complication, respectively, 7 years (patient 6) and 6 years (patient 12) after the first signs of scleroderma or atrophy. Patient 6 , who was diagnosed with LSCS at the left frontal area, presented with complex partial seizures in addition to complaints of hemicranial headache. Further investigations showed positive ANA not only in the serum but also in the CSF of this patient. 


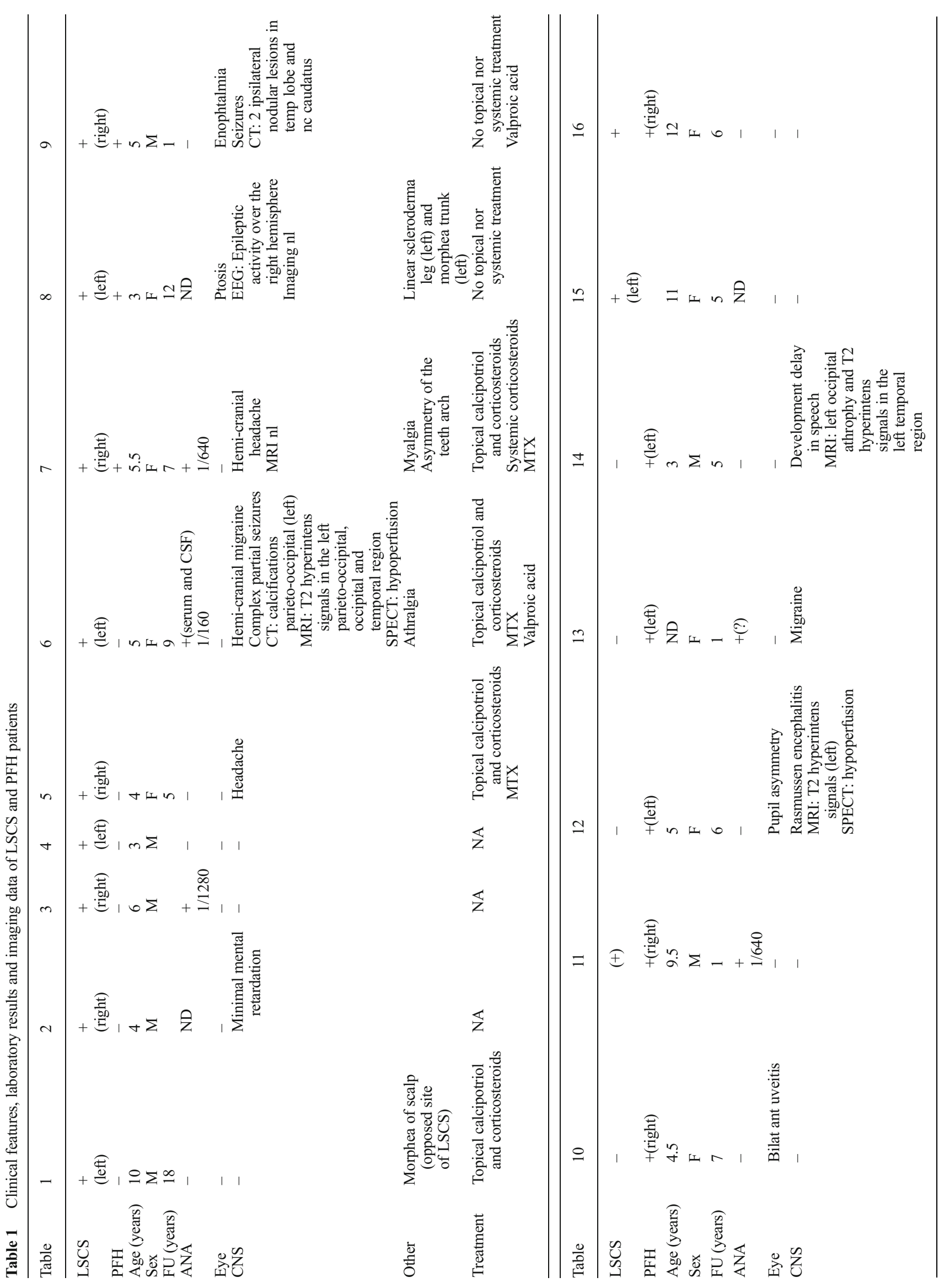




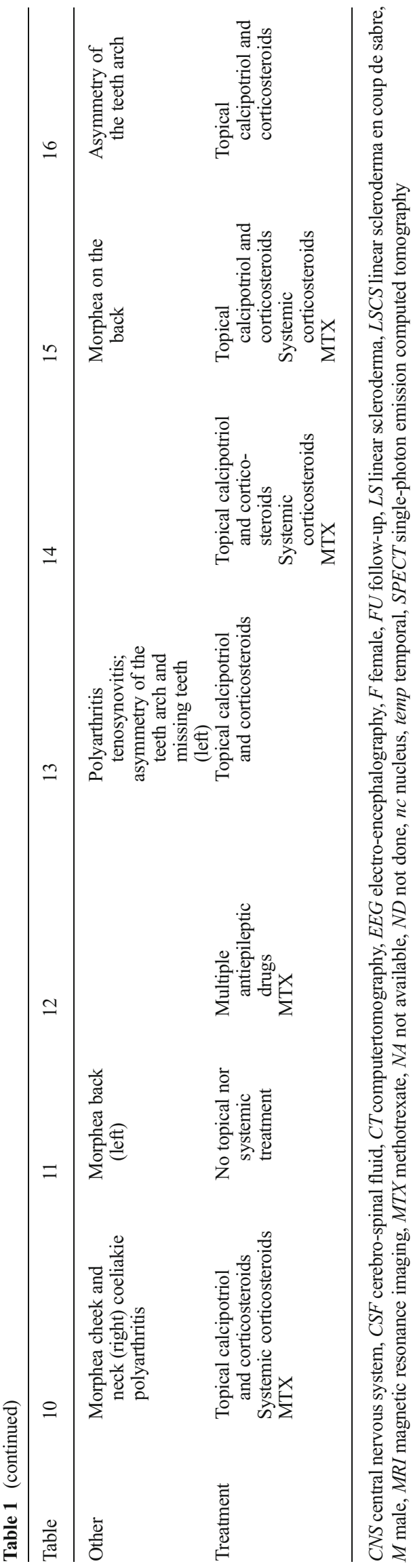

Initially, T2-hyperintense signals were seen on MRI in the white matter of the left parieto-occipital region, temporal and occipital region, the site of her LSCS lesion. Single photon emission computed tomography (SPECT) showed hypoperfusion in the temporoparietal region. She was treated with valproic acid and methotrexate. Her epilepsy was well controlled. After several years, lesions were less pronounced on MRI and SPECT perfusion images normalized (Fig. 1f). Patient 12, diagnosed with PFH, suffered from severe and progressive complex partial seizures becoming more generalized and continuous with time. They were associated with postictal hemiparesis and severe cognitive disturbances. MRI showed a T2-hyperintense signal in the left subcortical parietal area, corresponding to the site of her PRS and coinciding with a region of hypoperfusion on SPECT (Fig. 1g, h). With time, she developed new lesions on the left hemisphere and in the right parietal and frontal subcortical and cortical area. Brain biopsy showed an inflammatory infiltrate with neuronal damage consistent with Rasmussen encephalitis. Her epilepsy remained refractory to multiple anti-epileptic drugs and hemispherotomy. In the other 5 patients in whom MRI was performed, including 2 children with severe migraine, no abnormalities were found in 4 of them, except in patient 14 were T2-hyperintense signals were found in a child with a developmental delay. In patient 9, CT showed 2 ipsilateral nodular lesions in the temporal lobe and nucleus caudatus.

Two of our patients had polyarthritis (patients 10 and 13, diagnosed with PFH), mainly of the small joints. In one patient, wrist tenosynovitis was associated with a severe carpal tunnel syndrome.

Additional autoimmune features comprised uveitis and celiac disease in patient 10.

Of the 16 patients included, 8 patients were diagnosed before and 8 after the year 2000. There seemed to be no difference in age of onset of the disease and in both subpopulations; extracutaneous symptoms were equally present.

Laboratory tests showed presence of autoimmunity in the form of positive ANA in three out of nine LSCS patients and in two out of five patients with PFH. Only titers $>1 / 80$ were considered to be relevant. In patient 6 , ANA were found both in the serum and the CSF. In patient 12, an intrathecal IgG production was suggested/proven by the presence of nine oligoclonal IgG bands in the CSF without corresponding bands in the serum. During follow-up, additional analysis showed positive anti-NMDA receptor antibodies in the serum.

A skin biopsy performed in the region with hemiatrophy but without clinically visible sclerosis in patient 11 (with PFH) showed histopathological signs of fibrosis associated with a lymphocytic perivascular infiltrate and $\operatorname{IgG}$ and IgM deposits.

Children with only skin lesions received either topical steroids and calcipotriol or no treatment (if the lesions were stable). Patients 5, 6, 7, 10, 12, 14 and 15 were treated systematically with methotrexate with or without corticosteroids. In 


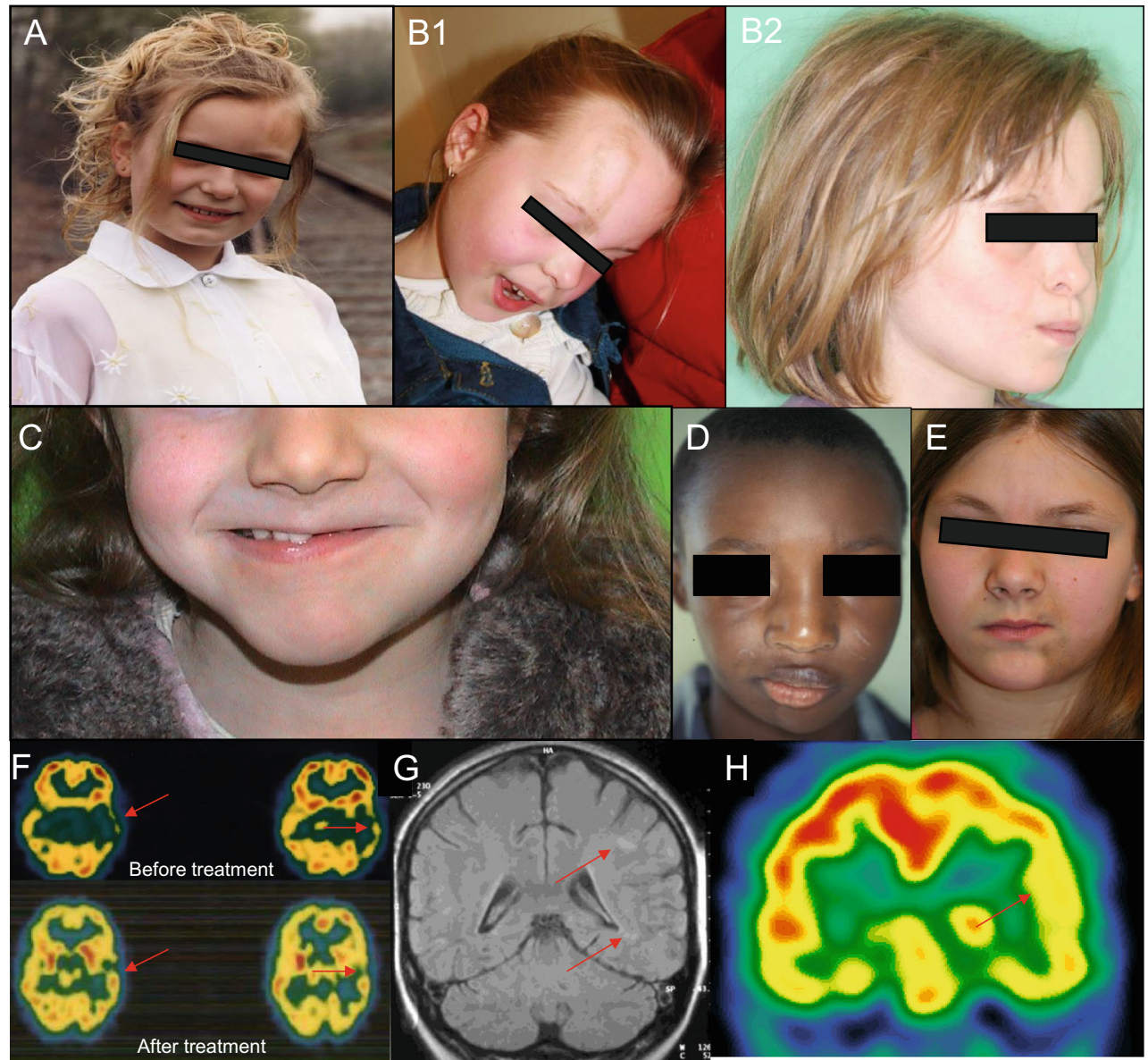

Fig. 1 Clinical pictures and imaging of some patients. a Patient 6: LSCS started at the age of 5. Lesions are present on the left forehead and frontal scalp with hair loss. b Patient 7: LSCS of the right forehead started at the age of 6 (1), at the age of 8 PFH of the right hemiface appeared (2). $\mathbf{c}$ Patient 10: 10-year-old girl with right hemifacial atrophy with morphea lesions of the right cheek and neck. d Patient 11: before PFH started, this boy had a small morphea lesion on his right forehead. Moreover, he had alopecia of the right frontotemporal area. e Patient 13: girl with PFH of the left side. She developed a small patch of morphea of the chin, hypoplasia of the maxillar bone and teeth anomalies. f Patient 6: LSCS patient with complex partial seizures. Initial hypoperfusion in the temperoparietal region on SPECT disappeared after treatment with MTX. $\mathbf{g}, \mathbf{h}$ MRI and SPECT of patient 12: area of hypoperfusion on SPECT (h) corresponds with hyperintense areas on T2 of MRI (g) patient 5, 6, 10, 14 and 15, this resulted in a remission of the clinical signs. In patient 7, the progression of the hemiatrophy could not be stopped. In patient 12, skin lesions stabilized, but neurologically, she deteriorated further (as described earlier).

\section{Discussion}

We present a large paediatric patient cohort of 16 children with LSCS and/or PFH, associated with autoimmunity and/or encephalitis. The clinical signs and onset of presentation were not different from what is known in literature for these conditions. Extracutaneous manifestations included dental, ophthalmological, autoimmune and neurological abnormalities. Our findings illustrate a wide clinical spectrum of these entities with significant overlap.
Our patient cohort corroborate the hypothesis that LSCS and PFH are similar clinical entities with LSCS and superficial skin involvement at one end of the spectrum and PFH with involvement of subcutaneous deep tissue at the other end, as suggested previously by Lehman et al. [10].

All patients had localized scleroderma lesions, nine of them had associated hemifacial atrophy. Four patients had been diagnosed with LSCS before atrophy of the ipsilateral face became apparent, which is in agreement with the findings of Tollefson [18]. In our cohort, most PFH patients had a small or larger lesion of localized scleroderma in the face. In one of our patients, the discrete scleroderma lesion had disappeared when his hemifacial atrophy was fully developed. The skin biopsy in our patient with clinical PFH showed histological signs typical for LSCS: fibrosis associated with a lymphocytic perivascular infiltrate and IgG and IgM deposits [9]. 
The spectrum of extracutaneous manifestations is similar for LSCS and PFH, but they seem to be more frequent in PFH. We observed dental complications in 3, ophthalmological in 4 and neurological complications in 9 of our patients. We also found the association with other autoimmune diseases (polyarthritis, celiac disease) in 2 of the 16 patients (1 with LSCS, 1 with the combination of LSCS and PFH). These findings are in agreement with a multicenter study by Zulian et al. in which neurological and ophthalmological complications were the most common extracutaneous manifestations in LSCS and PFH [21]. The most common ophthalmic manifestation of PFH and LSCS is progressive enophthalmia [6, 19], explained by atrophy of the orbital fat. The most common neurological complications are seizures and migraine, which may even be the presenting signs [16]. Most neurological problems are ipsilateral to the cutaneous signs (as in patient $6,7,8,14$ ); contralateral lesions have been described as well as generalized lesions, as in our patient 12 [18].

The pathogenesis of both LSCS and PFH is incompletely understood. Various proposed etiologies include trauma, infection, a disturbance of fat metabolism, a cranial vascular malformation or sympathic dysfunction [5]. More recently, an autoimmune aetiology has been proposed. By studying cytokine profiles in serum and skin biopsy specimens of scleroderma patients, Th1-responses seem to play an important role in the active state of the disease, whereas Th2responses are more important in fibrosis extent and disease damage. Th17-cells may be instrumental in the transition between these two phases as they can modulate the propagation of inflammation and the transition to the fibrotic state of the disease [9].

The neurological findings in two of our patients may strengthen the hypothesis of an autoimmune background for these two conditions. Autoimmune encephalitis is characterized by the presence of various autoantibodies both in serum and CSF of the affected individuals [13, 14]. In the two patients with the most severe neurological complications, additional autoimmune manifestations in the CSF were found: ANA in the CSF in one and signs of intrathecal IgG production with - several years after the initial presentation - the presence of anti-NMDA antibodies in the other. In the latter, the brain biopsy previously showed an inflammatory infiltrate with neuronal damage consistent with Rasmussen encephalitis. It is known that on immunofluorescent staining on biopsy specimens of Rasmussen patients, most of the infiltrating cells are $\mathrm{T}$ lymphocytes causing apoptotic death of neurons and astrocytes, but not of oligodendrocytes or myelin. Additional spectratyping revealed the clonal expansion of a subset of $\mathrm{CD}^{+} \mathrm{T}$ cells. Further, an accumulation of immunoglobulins and of $\mathrm{C} 3$ and $\mathrm{Clq}$ within the vessel wall can be found $[2,7]$. In Rasmussen encephalitis, MRI findings evolve with time. Initial imaging studies can be normal. The early stages are characterized with hyperintense signalling on T2 and FLAIR images in cortex and white matter, which seem to correlate well with the higher number of $\mathrm{T}$ cells and reactive astrocytes on histopathology (as was found in patient 12) [3]. Serial MRIs during the disease course can demonstrate progression, worsening of the hyperintense signalling and atrophy.

In cases of Parry-Romberg, white matter high signal intensity on MRI is a common neuroimaging finding. These white matter changes are considered as a slowly progressive inflammatory process, comparable with the pathophysiologic mechanisms of Ramussen's encephalitis [17].

Other cases of overlap between PFH and Rasmussen encephalitis have been reported $[13,14]$.

Linear scleroderma en coup de sabre/ progressive facial hemiatrophy

\begin{tabular}{|c|c|c|c|}
\hline \multirow{2}{*}{ 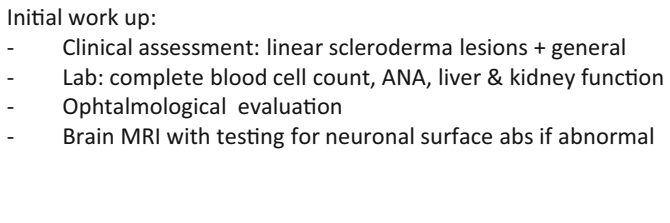 } & \multicolumn{3}{|c|}{$\begin{array}{c}\text { Topical calcitriol } \pm \text { corticosteroids } \\
\text { With/without } \\
\text { Systemic treatment }\end{array}$} \\
\hline & $\begin{array}{l}\text { Methrotrexate } \\
1 \mathrm{mg} / \mathrm{kg} / \text { week } \mathrm{PO} / \mathrm{SC} \\
\mathrm{max} 25 \mathrm{mg} \text { for } 1 \text { year }\end{array}$ & $\begin{array}{l}\text { Methrotrexate } 1 \mathrm{mg} / \mathrm{kg} / \text { week } \\
\mathrm{PO} / \mathrm{SC} \\
\max 25 \mathrm{mg} \text { for } 1 \text { year } \\
+\end{array}$ & $\begin{array}{l}\text { Methrotrexate } 1 \mathrm{mg} / \mathrm{kg} / \text { week } \\
\mathrm{PO} / \mathrm{SC} \\
\max 25 \mathrm{mg} \text { for } 1 \text { year } \\
+\end{array}$ \\
\hline $\begin{array}{l}\text { During follow-up: } \\
\text { - Clinical assessment: signs of active local disease. New } \\
\text { autoimmune manifestations (extracutaneous involvement) } \\
\text { - Lab for monitoring drug safety (complete bloof cell count, } \\
\text { liver \& kidney function) } \\
\text { - Ophtalmological evaluation once yearly } \\
\text { - Brain MRI if symptoms suggestive of neurological } \\
\text { involvement }\end{array}$ & & $\begin{array}{l}\text { Prednisone } 2 \mathrm{mg} / \mathrm{kg} / \text { day, } \max \\
60 \mathrm{mg} / \mathrm{d} \\
\text { Taper: } \\
\text { - } \text { Week 8: } 50 \% \\
\text { - } \text { Week } 16: 25 \% \\
\text { - } \text { Week 24: } 12,5 \% \\
\text { - } \text { Week } 48: \text { off corticosteroids }\end{array}$ & $\begin{array}{l}\text { Methylprednisolone } 30 \mathrm{mg} / \mathrm{kg} \\
\text { per dosis, max } 1 \mathrm{~g} \\
* 3 \text { consecutive days per } \\
\text { month for } 3 \text { months } \\
\text { OR } \\
\quad * 1 \text { dose/week for } 12 \text { weeks } \\
\text { No Taper }\end{array}$ \\
\hline
\end{tabular}

Fig. 2 Flow pathway illustrating proposed investigative (left) and management strategies (right) (according to Li et al. [11]). Decision to treat and choice of plan is based on physician judgment of best care. $P O$ per os, $S C$ subcutaneous 
Finally with immunosuppressive therapy (methotrexate and corticosteroids), an arrest of disease progression and stabilization of MRI findings could be obtained in some patients of our cohort.

There are at present no evidence-based guidelines about the optimal follow-up and treatment for LSCS and $\mathrm{PFH}$, as no large randomized studies are available. Many authors' advices to perform MRI in all patients with both LSCS and PFH as neurological complications are not seldom. Early signs may be detected by MRI [4] or even earlier by SPECT [12] before they become clinically relevant. How often MRI should be repeated and how long remain a matter of debate. Abnormalities on MRI or EEG have been described in patients without clinical neurological symptoms [18, 21], the significance over time is therefore uncertain. In patients with MRI/EEG abnormalities, we would recommend a systematic search for known autoantibodies to neuronal (surface) antigens (Fig. 2).

Most treatment options are based on immunosuppression. For localized scleroderma, a randomized therapeutic trial did demonstrate a beneficial effect of methotrexate in association with systemic steroids [20], but the efficacy of this combination in PFH has not been convincingly demonstrated. Some case reports suggest a positive effect of immunosuppressive therapy for PFH [8], but in other patients, those therapies did not prove to be helpful. Our cases treated with systemic corticosteroids and methotrexate seem to confirm those observations. For the patients treated in our institution from the time of diagnosis, we used a combination of steroids and methotrexate, as well as topical calcitriol, according to the guidelines published for juvenile localized scleroderma [11] (Fig. 2).

There is a clear need for a large prospective study in this group of patients to determine the risk factors for development of extracutaneous manifestations, adequate surveillance and therapy. A prospective study of natural history, immuneinflammatory characteristics and gene expression profile in a larger group of patients is essential to advance our understanding of disease course and pathogenesis, and to guide the development of adequate therapies.

\section{Conclusion}

Paediatricians taking care of children with LSCS and/or PFH should be aware of potential neurological involvement and other autoimmune manifestations. International collaborative efforts to study the natural history of this disease entity and to increase our understanding of an underlying immuneinflammatory pathogenesis may help develop more effective therapies.

Funding source No funding was received for this study.
Financial disclosure The authors have no financial relationships that could be relevant to the work.

Conflict of interest None of the authors have a conflict of interest.

Author's contributions Lien De Somer was responsible for the data collection and analysis from the three different hospitals, drafted the initial manuscript and approved the final manuscript as submitted. MarieAnne Morren conceptualized and designed the study, supervised the data collection and approved the final manuscript as submitted. Petra CE Hissink Muller and Karine Despontin critically collected data from the Dutch and Luxemburgh patients, respectively, reviewed the manuscript and approved the final manuscript as submitted. Lagae Lieven and Katrien Jansen supervised the data analysis for the paediatric neurology patients, reviewed the manuscript and approved the final manuscript as submitted. Wouters Carine designed the study, coordinated and supervised data collection, critically reviewed the manuscript and approved the final manuscript as submitted.

\section{References}

1. Armangue T, Petit-Pedrol M, Dalmau J (2012) Autoimmune encephalitis in children. J Child Neurol 27:1460-1469

2. Bien CG, Granata T, Antozzi C, Cross JH, Dulac O, Kurthen M, Lassmann H, Mantegazza R, Villemure JG, Spreafico R, Elger CE (2005) Pathogenesis, diagnosis and treatment of Rasmussen encephalitis: a European consensus statement. Brain 128:454-471

3. Bien CG, Urbach H, Deckert M, Schramm J, Wiestler OD, Lassmann H, Elger CE (2002) Diagnosis and staging of Rasmussen's encephalitis by serial MRI and histopathology. Neurology 58:250-257

4. Chiu YE, Vora S, Kwon EK, Maheshwari M (2012) A significant proportion of children with morphea en coup de sabre and ParryRomberg syndrome have neuroimaging findings. Pediatr Dermatol 29:738-748

5. El-Kehdy J, Abbas O, Rubeiz N (2012) A review of ParryRomberg syndrome. J Am Acad Dermatol 67:769-784

6. Holland KE, Steffes B, Nocton JJ, Schwabe MJ, Jacobson RD, Drolet BA (2006) Linear scleroderma en coup de sabre with associated neurologic abnormalities. Pediatrics 117:e132-e136

7. Kim SJ, Park YD, Hessler R, Lee MR, Smith JR (2010) Correlation between magnetic resonance imaging and histopathologic grades in Rasmussen syndrome. Pediatr Neurol 42:172-176

8. Korkmaz C, Adapinar B, Uysal S (2005) Beneficial effect of immunosuppressive drugs on Parry-Romberg syndrome: a case report and review of the literature. South Med J 98:940-942

9. Kurzinski K, Torok KS (2011) Cytokine profiles in localized scleroderma and relationship to clinical features. Cytokine 55:157-164

10. Lehman TJ (1992) The Parry Romberg syndrome of progressive facial hemiatrophy and linear scleroderma en coup de sabre. Mistaken diagnosis or overlapping conditions? J Rheumatol 19: 844-845

11. Li SC et al (2012) Development of consensus treatment plans for juvenile localized scleroderma: a roadmap toward comparative effectiveness studies in juvenile localized scleroderma. Arthritis Care Res (Hoboken) 64:1175-1185

12. Mazzeo N, Fisher JG, Mayer MH, Mathieu GP (1995) Progressive hemifacial atrophy (Parry-Romberg syndrome). Case report. Oral Surg Oral Med Oral Pathol Oral Radiol Endod 79:30-35

13. Paprocka J, Jamroz E, Adamek D, Marszal E, Mandera M (2006) Difficulties in differentiation of Parry-Romberg syndrome, unilateral facial sclerodermia, and Rasmussen syndrome. Childs Nerv Syst 22:409-415 
14. Shah JR, Juhasz C, Kupsky WJ, Asano E, Sood S, Fain D, Chugani HT (2003) Rasmussen encephalitis associated with Parry-Romberg syndrome. Neurology 61:395-397

15. Sommer A, Gambichler T, Bacharach-Buhles M, von Rothenburg T, Altmeyer P, Kreuter A (2006) Clinical and serological characteristics of progressive facial hemiatrophy: a case series of 12 patients. J Am Acad Dermatol 54:227-233

16. Stone J (2003) Parry-Romberg syndrome: a global survey of 205 patients using the Internet. Neurology 61:674-676

17. Terstegge K, Kunath B, Felber S, Speciali JG, Henkes H, Hosten N (1994) MR of brain involvement in progressive facial hemiatrophy (Romberg disease): reconsideration of a syndrome. AJNR Am J Neuroradiol 15:145-150

18. Tollefson MM, Witman PM (2007) En coup de sabre morphea and Parry-Romberg syndrome: a retrospective review of 54 patients. J Am Acad Dermatol 56:257-263
19. Zannin ME, Martini G, Athreya BH, Russo R, Higgins G, Vittadello F, Alpigiani MG, Alessio M, Paradisi M, Woo P, Zulian F (2007) Ocular involvement in children with localised scleroderma: a multi-centre study. Br J Ophthalmol 91:1311-1314

20. Zulian F, Martini G, Vallongo C, Vittadello F, Falcini F, Patrizi A, Alessio M, La TF, Podda RA, Gerloni V, Cutrone M, BelloniFortina A, Paradisi M, Martino S, Perilongo G (2011) Methotrexate treatment in juvenile localized scleroderma: a randomized, double-blind, placebo-controlled trial. Arthritis Rheum 63:1998-2006

21. Zulian F et al (2005) Localized scleroderma in childhood is not just a skin disease. Arthritis Rheum 52:2873-2881

22. Zulian F et al (2006) Juvenile localized scleroderma: clinical and epidemiological features in 750 children. An international study. Rheumatology (Oxford) 45:614-620 